Research Article

\title{
Percutaneous Kyphoplasty Guided by CT Images Based on SEPB Algorithm in the Treatment of Elderly Osteoporotic Thoracolumbar Vertebral Compression Fractures
}

\author{
Yanming He $\mathbb{D}^{1,2}$ Shujun Zhang $\mathbb{D}^{1,2}$ Xueguang Liu $\mathbb{D}^{1},{ }^{2}$ Dong Mao $\mathbb{D}^{\mathbb{D}}, 2$ \\ and Zhenzhong Sun $\mathbb{1}^{2}$ \\ ${ }^{1}$ Soochow University, Suzhou 215008, Jiangsu Province, China \\ ${ }^{2}$ Spinal Surgery, Wuxi No. 9 Hospital Affiliated to Soochow University, Wuxi 214000, Jiangsu Province, China \\ Correspondence should be addressed to Zhenzhong Sun; 2006020128@st.btbu.edu.cn
}

Received 6 May 2021; Accepted 13 July 2021; Published 24 July 2021

Academic Editor: Gustavo Ramirez

Copyright (C) 2021 Yanming He et al. This is an open access article distributed under the Creative Commons Attribution License, which permits unrestricted use, distribution, and reproduction in any medium, provided the original work is properly cited.

\begin{abstract}
The paper uses the SEPB algorithm to explore the value of X-ray and CT diagnosis in elderly patients with osteoporotic lumbar compressive fractures, while observing percutaneous kyphoplasty (PKP) in the treatment of elderly osteoporotic compression fractures with clinical efficacy. 38 elderly patients with fractured osteoporotic compression fractures who came to our hospital for treatment were included. All patients were diagnosed by X-ray and CT, the clinical data of all patients were analyzed, the imaging findings related to X-ray and CT diagnosis were clarified, and the diagnostic coincidence rate was analyzed. At the same time, PKP treatment was applied for clinical efficacy and Imaging analysis. And, follow-up was conducted for 2 months after operation. The results showed that compared with the X-ray diagnosis, the accuracy of CT diagnosis was $88.89 \%$ (32/38), and the difference between the groups was significant $(P<0.05)$. 35 cases of low back pain disappeared after operation, and 3 cases of pain were significantly reduced without bone cement leakage. Postoperative imaging examination showed no space occupied in the spinal canal, and kyphosis deformity was significantly improved. The average height of the anterior vertebral column after injury was significantly increased $(P<0.05)$. The Cobb angle returned to normal level, which was statistically significant compared with that before the operation $(P<0.05)$. In conclusion, in the diagnosis of elderly patients with osteoporotic lumbar compression fractures, the coincidence rate of CT diagnosis is better than that of X-ray diagnosis. Therefore, the application rate of CT diagnostic methods in diagnosis is higher, which provides an effective basis for clinical diagnosis and treatment. PKP surgery is less invasive, safe, and has good clinical efficacy. It can quickly relieve pain and effectively restore the height of injured vertebrae. It is an ideal treatment method for elderly osteoporotic thoracolumbar vertebral compression fractures.
\end{abstract}

\section{Introduction}

Nowadays, the trend of aging in China is getting worse, and osteoporosis has a higher incidence. Therefore, the incidence of osteoporotic lumbar compression fractures in elderly patients has increased significantly. Osteoporotic vertebral compression fractures are a common condition of spinal fractures in the elderly. Fractures are the most common and serious complication of osteoporosis. There are approximately 70,000 osteoporotic vertebral compression fractures each year in the United States, and $20 \%$ of patients have secondary fractures within 1 year. Among them, 2/3 of
OVCFs have no obvious clinical symptoms, but still $1 / 3$ of fractures will be combined with pain, kyphosis, dysfunction, and other sequelae, which seriously affect the quality of life of patients. The main symptoms are pain, restricted mobility, and secondary dysfunction in multiple systems such as breathing, digestion, and cardiovascular disease, which are important causes of death in patients with osteoporosis. The traditional treatment method is long-term bed rest plus drug nonsurgical treatment. However, long-term bed rest is likely to lead to increased osteoporosis and complications; surgical treatment due to osteoporosis has a poor reduction and fixation effect and is also prone to surgical complications. Percutaneous 
kyphoplasty (PKP) restores the height of the compressed vertebral body through the expansion of the balloon and strengthens the vertebral body by injecting bone cement to restore its anatomical shape and mechanical properties, achieving minimally invasive, rapid analgesia and early activity. The purpose of treatment is to provide the premise and basis for the subsequent medical treatment of osteoporosis.

MRI diagnosis technology is of great value in the diagnosis of elderly patients with osteoporotic lumbar compressive fracture disease. This kind of examination method can effectively confirm the diagnosis of elderly patients with osteoporotic lumbar compressive fracture disease. However, MRI diagnosis technology has a high price, and a small number of patients will not be able to accept this diagnosis because of the price, so there are certain limitations in clinical diagnosis. At present, CT and X-ray diagnostic methods are widely used in the diagnosis of elderly patients with osteoporotic lumbar compression fractures, and their diagnostic value is now studied. From July 2017 to May 2018, we used PKP to treat 38 cases of elderly osteoporotic compression fractures and achieved good clinical efficacy. The reports are as follows [1].

\section{General Information and Methods}

2.1. General Information. All cases in this study were inpatients in the hospital from March 2018 to March 2020. 38 cases met the inclusion criteria. There were 13 males and 25 females. The age ranged from 58 to 85 years, with an average of 71.2 years. 29 cases were injured by falls, 6 cases were caused by carrying heavy objects, and 3 cases were caused by other causes. There were 5 cases of T10 vertebral body, 6 cases of T11 vertebral body, 12 cases of T12 vertebral body, 7 cases of L1 vertebral body, 5 cases of L2 vertebral body, and 3 cases of L3 vertebral body. There were 5 cases of cardiovascular system disease, 1 case of urinary system disease, and 3 cases of endocrine system disease. According to Jikei's osteoporosis classification standard, 5 cases were in the early stage (the number of trabecular bone is normal, the bone density is reduced, and the trabecular bone is thinned); Grade I: decreased transverse trabecular bone, vertical trabecular bone, and endplate protrusion. Grade II: transverse trabecular bone reduced further, and vertical bone trabecula also decreased. Grade III: transverse trabecular bone nearly disappeared, and vertical bone trabecula also not clear, shaped like grass. The study was approved by the ethics committee of hospital, and informed consent was signed by each participant. The study was approved by the ethics committee of hospital, and informed consent was signed by each participant.

2.2. Case Selection Criteria. Inclusion criteria: the "Guidelines for the Diagnosis and Treatment of Osteoporotic Fractures" issued by the Osteoporosis and Bone Mineral Disease Branch of the Chinese Medical Branch is referred. (1) The elderly (>50 years old); (2) minor trauma or no history of obvious trauma; (3) persistent pain in the chest or waist cannot be turned over, unable to move, and cannot be forced; (4) stiff chest or lumbar spine; (5) there is a tender point on the chest or waist; (6) bone mineral density dualenergy X-ray absorption method (DXA) shows that the degree of bone mineral density reduction is equal to and greater than 2.5 standard deviations ( $T \geq-2.5 \mathrm{SD}$ ); (7) X-ray or the CT film showed that the front of a vertebral body in the chest or lumbar flattened, and the bone was broken, in a wedge shape. Diagnosis criteria (2) (5) with more than one abnormality, plus (1), (6), and (7) can be diagnosed as osteoporotic compression fracture. The standard of included cases were as follows: permit systemic conditions, without serious diseases of important organs such as heart, brain, and lung, continue prone for 1 to 2 hours; the posterior wall of the vertebral body must be intact to prevent expansion of surrounding structures due to balloon compression and displace the bone mass or mass to cause or increase the risk of compression; CT, MRI, bone density test, and other imaging examinations show osteoporotic compression fractures. Exclusion criteria: (1) the vertebral body is severely compressed and the guide needle and infusion agent cannot be placed, or the middle column of the vertebral body is destroyed, and the spinal cord is compressed and (2) severe cardiopulmonary disease cannot tolerate surgery or hemorrhagic disease.

\subsection{Method}

\subsubsection{Surgical Methods}

(1) Related Equipment. The surgical instruments are produced by Shandong Guanlong Medical Products Co., Ltd. The complete set of instruments includes puncture needle $(\Phi 4.0 \times 126 \mathrm{~mm})$, balloon $(\Phi 12 \times 19 \mathrm{~mm}$ after inflation), surface pressure intensifier $(20 \mathrm{~mL} / 30 \mathrm{~atm})$, bone cement injection device $(\Phi 3.4 \times 195 \mathrm{~mm})$, solid core vertebral body drill $(\Phi 3.4 \times 190 \mathrm{~mm})$, hollow core vertebral body drill $(\Phi 3.4 \times 190 \mathrm{~mm})$, and push $\operatorname{rod}(\Phi 2.0 \times 220 \mathrm{~mm})$. The filling material is polymethyl methacrylate (PMMA) bone cement.

(2) Preoperative Preparation. Routine physical examination and laboratory examination, chest radiograph, electrocardiogram, and lung function examination are carried out to actively treat complications so that the patient's general condition is in a state suitable for surgical treatment. X-rays of the affected area are taken, and if necessary, CT and MRI are performed to determine the location of the lesion and the nature of the lesion and have a full understanding of the fracture situation and vertebral body stability.

(3) Surgical Methods. The patient was placed in the prone position, the diseased vertebrae were aligned with the center of the operating table, and the C-arm X-ray machine was used to position the body surface under fluoroscopy. Routine disinfection, towel laying, and local anesthesia with $1 \%$ lidocaine were carried out. Skin was cut with an incision about $0.5 \mathrm{~cm}$. Under the perspective guidance of the $\mathrm{C}$-arm $\mathrm{X}$-ray machine in the lateral position, the cored puncture needle is directly passed behind the vertebral body through the diseased pedicle. When the puncture needle reaches the 
posterior $1 / 3$ of the vertebral body, a ring saw is used to take the specimen; to the junction of the anterior $1 / 3$ and posterior $2 / 3$ of the vertebral body, the vertebral body drill is removed, the balloon is inserted through the working sleeve, and the belt is used. The pressure inflator gradually expands the balloon and injects the developer. At any time, attention is paid to check whether the pressure in the balloon drops. The pressure of the balloon system should be controlled below 16 atmospheres. After achieving satisfactory results of vertebral body expansion, the balloon catheter is removed, and the bone cement injector prefilled with bone cement is sent through the working sleeve to the anterior middle of the cavity of the vertebral body under perspective, and the bone cement is injected into the vertebral body $1.5 \sim 2.5 \mathrm{~mL}, \mathrm{C}$-arm $\mathrm{X}$-ray machine in the lateral fluoroscopy confirms that no bone cement oozes out of the vertebral body and waits for the bone cement to solidify, and then exits the bone cement filler and working sleeve, and the incision is sewed. After 10 minutes of observation, both lower limbs were moving normally and vital signs were stable and then returned to the ward. The operation flow chart is shown in Figure 1. After bed rest for 6 to 12 hours, he can walk down the ground, routinely apply antibacterial drugs for 3 days, perform spine $\mathrm{X}$-ray radiography and CT scan, check for bone cement overflow, and compare it with preoperative X-ray film to observe the injured vertebra, the average height of the center column, and the Cobb angle.

2.3.2. X-Ray and CT Diagnosis. All patients were diagnosed by X-ray and CT. X-ray diagnosis mainly diagnosed the patient's lumbar spine with orthophoto and lateral radiographs. CT diagnosis is as follows: the patient is kept in a supine position, the fractured vertebral body is scanned, the layer thickness is set to $2.0 \mathrm{~mm}$, the layer distance is set to $2.0 \mathrm{~mm}$, the scanning direction is parallel to the upper and lower edges of the vertebral body, and the bone window and mediastinum window are observed. The X-ray and CT imaging performance is analyzed to understand the diagnostic coincidence rate.

2.4. Imaging Judgment Index. All patients were diagnosed by $\mathrm{X}$-ray and CT, the clinical data of all patients were analyzed, the imaging findings related to X-ray and CT diagnosis were clarified, and the diagnostic coincidence rate was analyzed.

2.5. Efficacy Evaluation and Follow-Up. The visual analogue scale (VAS) of the pain method was used to assess the change in pain before and after the operation. A score of 10 was the most painful and 0 was no pain. The X-ray film of the thoracolumbar spine was taken to understand the loss of vertebral height and fracture healing. The reduction of the injured vertebrae was evaluated by calculating the change of the height of the anterior column of the injured vertebrae and the change of the Cobb angle before and after the operation. Through the comparative analysis of the above indicators, the efficacy of PKP in the treatment of OVCFs was evaluated and followed up for 2 months after operation [2].
2.6. Statistical Analysis. SPSS16.0 statistical software package is used. All observation indicators are represented by “ $\bar{x} \pm s$," using $t$-test. Inspection level $\alpha=0.05$. The probability that the high-level node and the low-level node are selected as cluster heads is $p_{m}$ and $p_{k}$, as shown in Figure 2 for the SEP algorithm flow [3]:

$$
\begin{aligned}
p_{m} & =\frac{p}{1+\alpha u}(1+\alpha), \\
p_{k} & =\frac{p}{1+\alpha u} .
\end{aligned}
$$

Among them, $p$ represents the proportion of cluster heads. The thresholds of high-level nodes and low-level nodes are as follows:

$$
\begin{gathered}
T\left(n_{m}\right)= \begin{cases}\frac{p_{m}}{1-p_{m}\left[\operatorname{rmod}\left(1 / p_{m}\right)\right]}, & n_{m} \in G_{1}, \\
0, & \text { others, }\end{cases} \\
T\left(n_{k}\right)= \begin{cases}\frac{p_{k}}{1-p_{k}\left[r \bmod \left(1 / p_{k}\right)\right]}, & n_{m} \in G_{2}, \\
0, & \text { others. }\end{cases}
\end{gathered}
$$

Before the election of the district leader, all nodes in the network broadcast and communicate according to the competition radius in formula (3) and summarize the neighbor node information of each node. The formula for the radius of competition is as follows:

$$
R_{i}=\left(1-c \frac{d_{\max }-d(i, \mathrm{BS})}{d_{\max }-d_{\min }}\right) R_{\max } .
$$

Among them, $c$ is taken as $0.5 ; d_{\max }$ and $d_{\min }$ are the maximum and minimum distances of all nodes in the network to the sink node; $d(i, \mathrm{BS})$ represents the distance from node $i$ to the sink node; and $R_{\max }$ is the maximum competition radius of the node. In the P-SEP district-first election algorithm, node $i$ participates in the election time:

$$
t_{i}= \begin{cases}k \times T_{\mathrm{CH}} \times \frac{n_{\mathrm{avg}}}{n_{i}}, & r=1, \\ k \times T_{\mathrm{CH}} \times\left(\frac{e_{i}^{r-1}}{e_{\mathrm{avg}}^{r-1}}+\frac{n_{\mathrm{avg}}}{n_{i}}\right), & r>1 .\end{cases}
$$

Among them, $k$ is a random number evenly distributed between $(0,0.1)$ to prevent two nodes from participating in the election at the same time; $T_{\mathrm{CH}}$ is the time required for the predefined district head election; $e_{\text {avg }}^{r-1}$ represents all nodes in round $r-1$ which is the average value of energy consumption; $e_{i}^{r-1}$ represents the energy consumption value of node $i$ in the $r-1$ round of the election node; $n_{i}$ represents the number of neighbor nodes of node $i$; and $n_{\text {avg }}$ is the average of the number of neighbor nodes. According to formula (4), the election time of node $i$ depends on the energy consumption of nodes and 


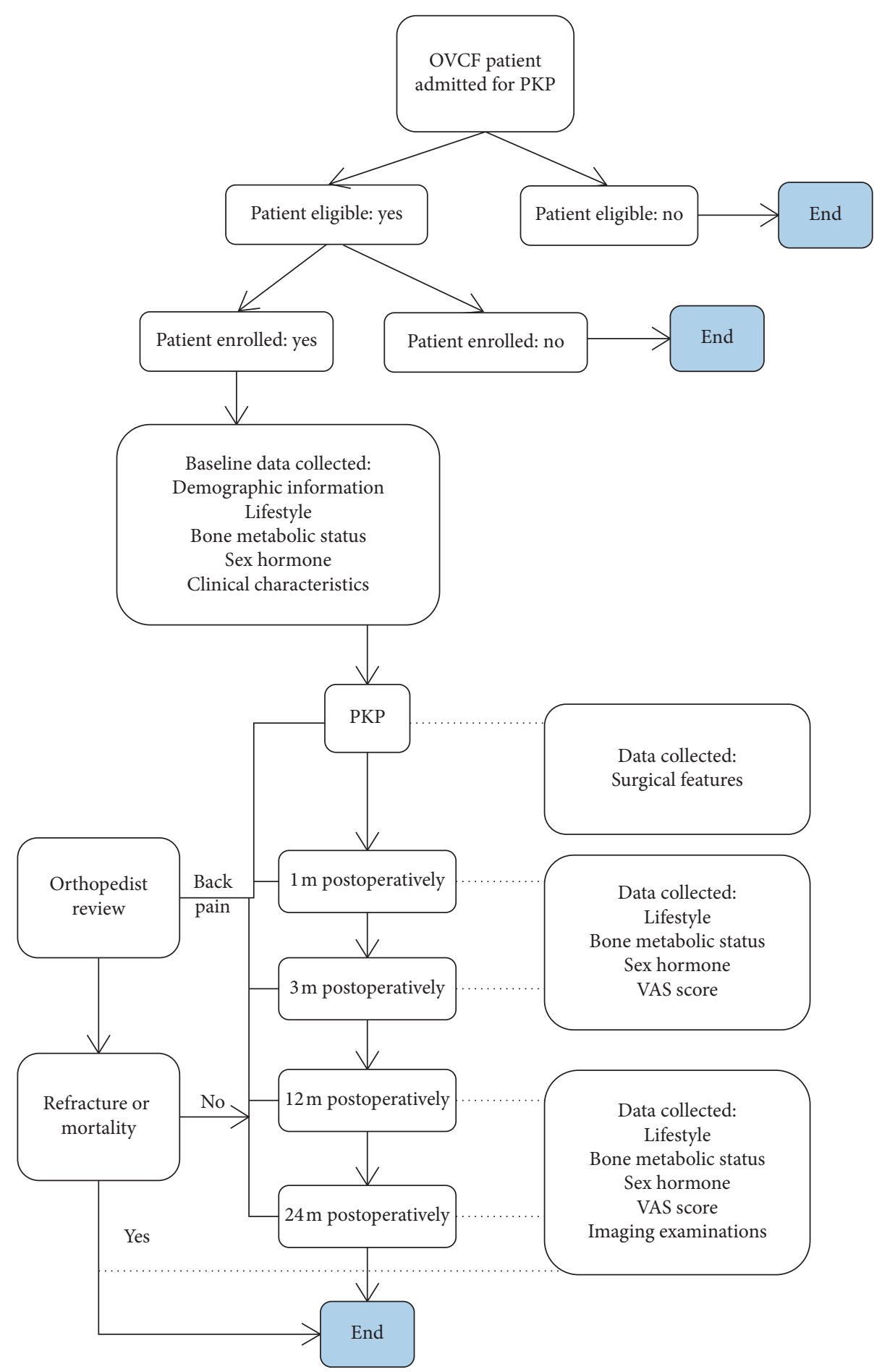

FIGURE 1: PKP treatment of osteoporotic thoracolumbar compression fractures in the elderly.

other nodes, the number of neighbor nodes, and the number of neighbor nodes of other nodes.

\section{Results}

3.1. Surgical Treatment Results. All 38 patients had successful operations, with an average operating time of 60 minutes and a hospital stay of 4-10 days, with an average of 7 days.
There were no intraoperative deaths and acute adverse reactions of the cardiovascular and cerebrovascular systems. After operation, the pain in the lower back was completely relieved in 35 cases, and 3 cases were significantly reduced. There was no leakage of bone cement. Except for 8 cases who complained of soreness in the chest and waist on the day of surgery, no other discomfort symptoms and complications occurred. There were statistically significant differences 


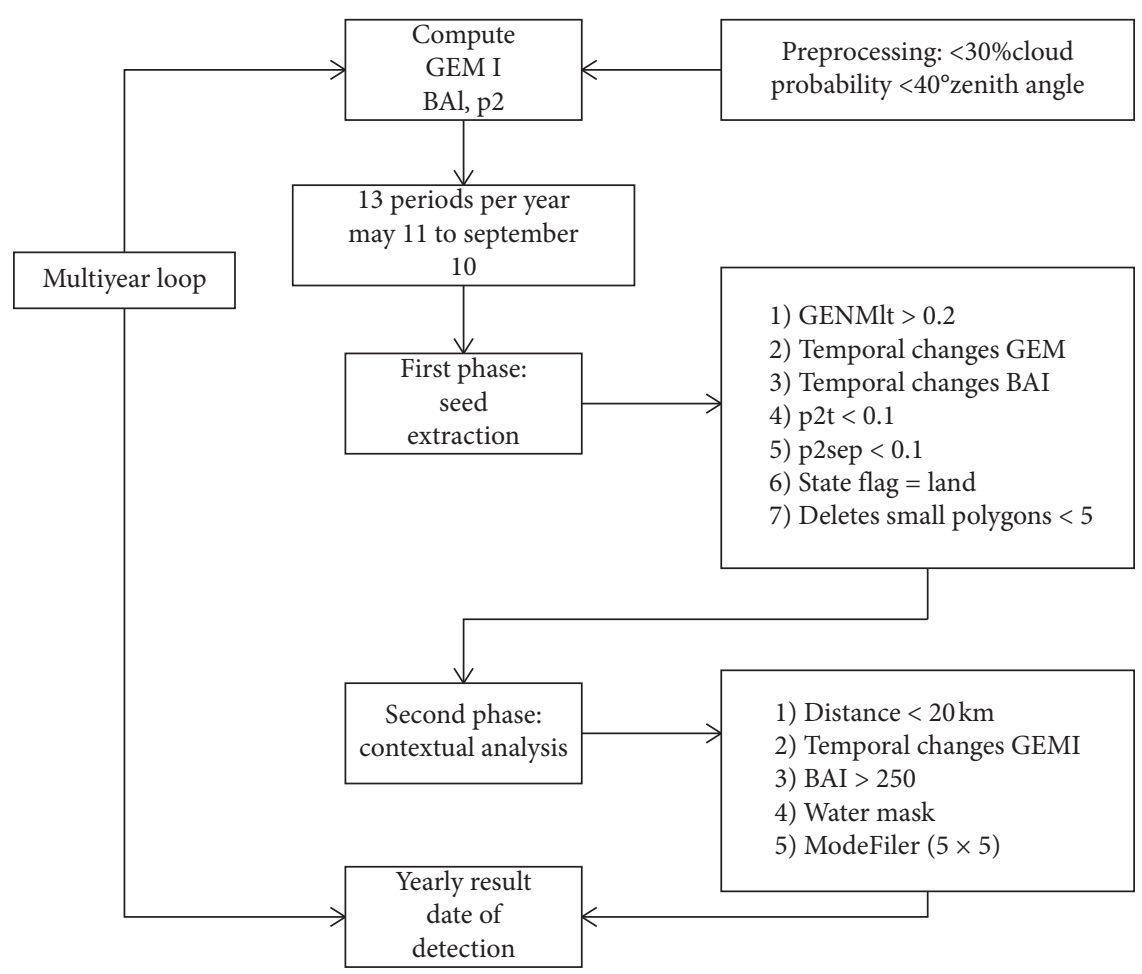

FIGURE 2: SEP algorithm process.

between the two postoperative scores, the average height of the injured vertebrae, the middle column, and the Cobb angle before the operation $(P<0.05)$, as shown in Table 1 . The X-ray film of the thoracolumbar segment taken 2 months after surgery showed that the fracture line was blurred, and there was continuous callus passing through the fracture line, indicating that the patient's thoracolumbar vertebral fracture had reached the clinical fracture healing standard [4].

3.2. CT and X-Ray Results. Compared with the X-ray diagnosis results, the accuracy of CT diagnosis was $88.89 \%$ (32/ 38 ), and the difference between the groups was significant $(P<0.05)$. Symptom analysis of imaging diagnosis of osteoporotic lumbar compressive fracture is shown in Table 2.

$X$-Ray Diagnosis. The compressed vertebral body is mainly wedge-shaped, flat, double-concave, and other shapes, and the double-concave shape is very typical. Compression of the posterior superior corner of the vertebral body toward the spinal canal is called the characteristic symptom of fracture. The bone density of the lumbar spine decreased significantly, and the cortical bone gradually became thinner and arranged in a grid-like arrangement, belonging to the longitudinal trabecular bone, as shown in Figure 3 for the X-ray imaging.

CT Diagnosis. The cortical bone in the anterior center column of the compressed vertebral body was obviously interrupted, and there was or was not a fracture line. It is stenotic, with reduced trabecular bone texture and thinner cortical bone. Figure 4 shows the CT imaging of osteoporotic thoracolumbar compression fractures in the elderly.

\section{Discussion}

4.1. PKP Technology Treatment. Osteoporosis is a skeletal metabolic disorder characterized by reduced bone mass and increased fracture risk, including bone mineralization disorders, bone calcium dissolution, decreased bone density, trabecular bone thinning, and easy to break. The strength of the bone decreases, the brittleness increases, and it is difficult to bear the original load. The more serious complication is vertebral compression fractures caused by osteoporosis. Common fractures include spine, hip, wrist, and shoulder fractures, of which spine compression fractures are the most common. Osteoporotic vertebral compression fractures are not easy to reduce and difficult to fix and often have low back pain as the main symptom. Most patients are elderly and often have various medical diseases. Osteoporosis is very common for the elderly. It is a common degenerative disease of bone tissue. Both bone mass and bone quality have significant changes, and it is easy to cause compression fractures. Among them, fractures of lumbar vertebra compression are very common. For elderly patients with osteoporotic lumbar compressive fractures, if they cannot maintain bed rest and actively undergo surgery and medical treatment, the clinical symptoms of the patients are significantly worsened, and there is a certain degree of motor dysfunction, and other complications also seriously affect the patient's health and quality of life. Therefore, it is of high value to diagnose the osteoporotic lumbar vertebral compression fracture disease as early as possible [5]. 
TABLE 1: Comparison of VAS scores of average heights of anterior vertebrae, middle column, and Cobb angle before and after operation $(\bar{x} \pm s, n=38)$.

\begin{tabular}{|c|c|c|c|}
\hline Detection indicator & Preoperative & Postoperative & 2 months after surgery \\
\hline Injured anterior column height (mm) & $15.1 \pm 1.9$ & $4.2 \pm 2.3$ & $4.4 \pm 1.9$ \\
\hline Cobb angle $\left({ }^{\circ}\right)$ & $21.3 \pm 4.2$ & $10.2 \pm 3.3$ & $10.1 \pm 3.2$ \\
\hline VAS score (points) & $8.3 \pm 1.1$ & $2.9 \pm 1.2$ & $2.1 \pm 1.0$ \\
\hline
\end{tabular}

TABle 2: Comparison of the coincidence rate of X-ray and CT diagnosis (cases/\%).

\begin{tabular}{|c|c|c|}
\hline Group & Number of cases & Coincidence rate $(\%)$ \\
\hline $\mathrm{X}$-ray $(n=38)$ & 20 & 55.56 \\
\hline CT $(n=38)$ & 32 & 88.89 \\
\hline$\chi^{2}$ value & \multicolumn{2}{|c|}{9.9692} \\
\hline$P$ value & & \\
\hline
\end{tabular}

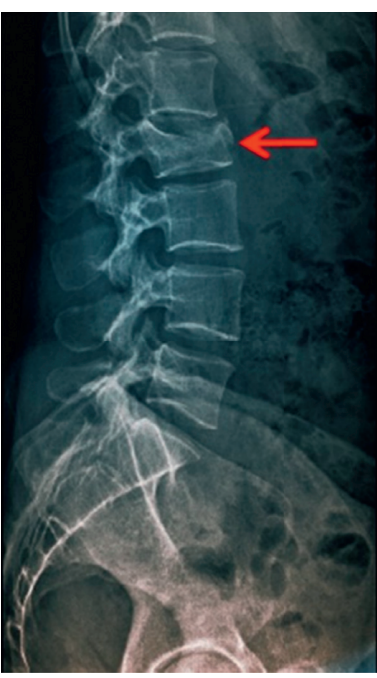

(a)

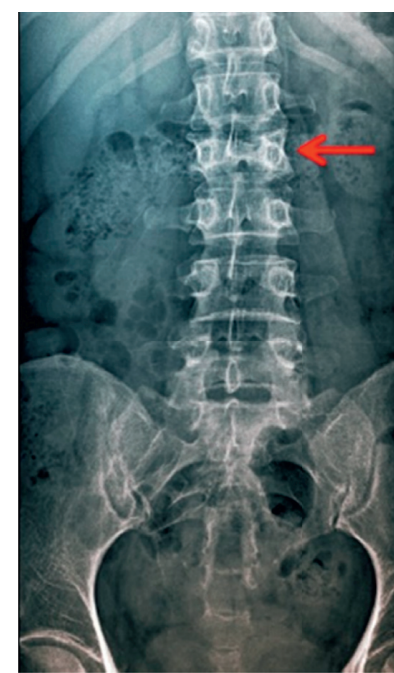

(b)

FIGURE 3: X-ray imaging of osteoporotic thoracolumbar vertebral compression fractures in the elderly.

The principle of PKP technology is as follows. It is to insert the balloon into the compressed vertebral body along the percutaneously inserted cannula and inflate it by injecting a contrast agent to form a cancellous bone cavity. The height of the vertebral body was restored, and bone cement was injected into the cavity. Because PKP is injecting the bone cement into the cavity formed, the injection pressure is low, the fluidity is low, the leakage of bone cement is significantly reduced, and the safety is higher. Due to the recovery of shape and height of the injured vertebral, the correction of deformity, the vertebral body is stabilized after the bone cement is solidified, so the pain can disappear immediately. The efficacy of this group of patients also confirms this. The VAS score before and after the operation, the height of the injured vertebrae, and the Cobb angle of the spine are statistically significant $(P<0.05)$.

PKP is suitable for the pain caused by collapse and destruction of the vertebral body, and the posterior wall of the vertebral body is relatively complete. Its indications include osteoporotic vertebral body compression fracture, vertebral body hemangioma, vertebral body metastasis tumor, and multiple myeloma of the vertebral body. In general, fresh fractures have better pain relief effects than older fractures, and the analgesic effect on osteoporotic fractures is better than vertebral tumors. Single vertebral fractures have more obvious pain relief than multiple fractures. It is a relatively contraindication for patients to be on anticoagulant therapy because the use of drugs to maintain anticoagulant status can increase the risk of surgery and prolong hospital stay. Acute infections of the skin of the whole body or the local area of surgery and chronic infections such as vertebral tuberculosis and vertebral osteomyelitis are absolute contraindications to PKP. Most of the complications of PKP are related to bone cement. Most clinical cases report asymptomatic bone cement leakage rates ranging from $10 \%$ to $15 \%$. Symptomatic complications are often related to the systemic consequences of bone cement flowing backward from the vertebral body into the spinal canal or intervertebral foramen or forward into the paravertebral venous plexus. 

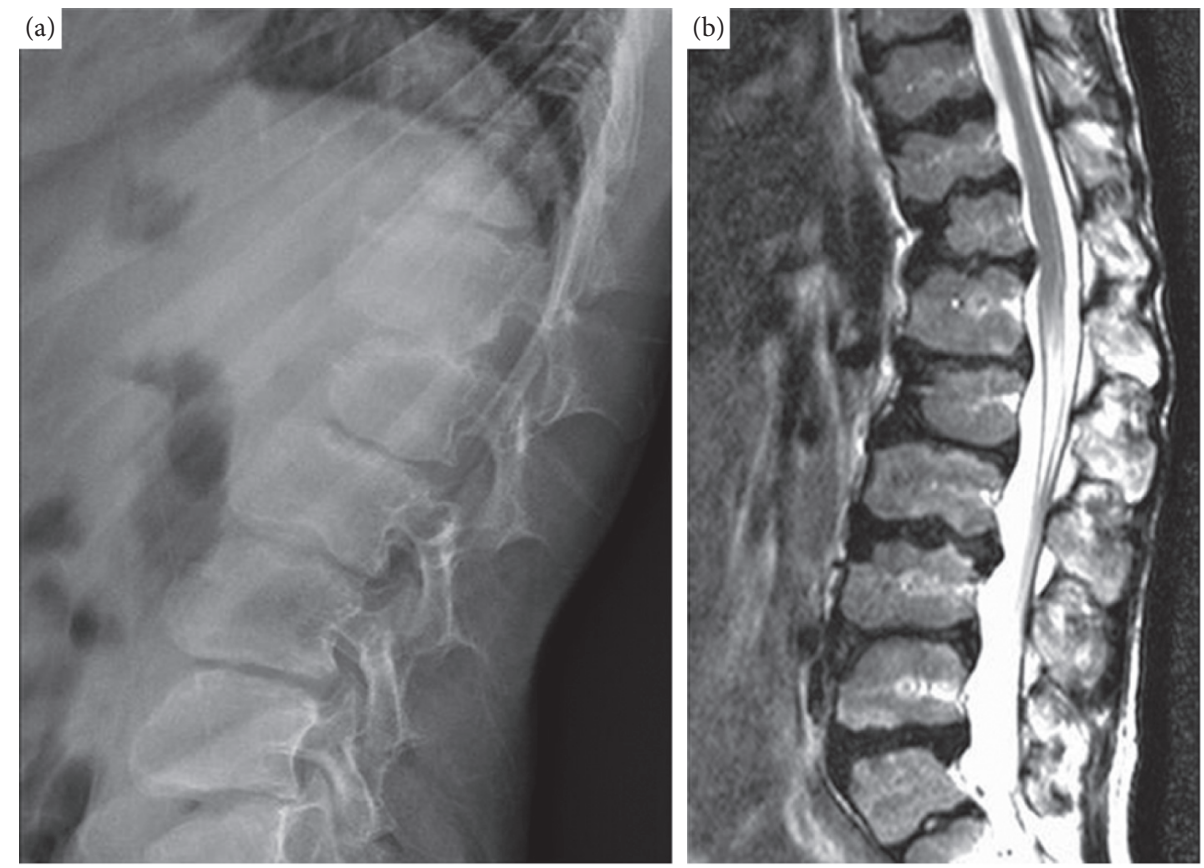

FIGURE 4: CT imaging of osteoporotic thoracolumbar vertebral compression fractures in the elderly.

4.2. Compare the Coincidence Rate of X-Ray and CT Diagnosis. $\mathrm{X}$-ray diagnosis mainly uses rays to penetrate the location of the lesion to form a two-dimensional image. It is an imaging diagnostic method. It is an important diagnostic method for lumbar fracture disease. It has the advantages of simple operation and economical price. However, this method is not very obvious for the fracture of the posterior vertebral body and the vertebral arch. There are limitations in the diagnosis of osteoporotic lumbar vertebral compression fractures in the elderly. Compared with X-ray diagnosis, CT diagnosis has higher resolution and clearer image. It can observe the window changes and judge the vertebral body, paravertebral tissue and spinal canal, and then find that the $\mathrm{X}$-ray cannot show the fracture.

\section{Conclusion}

In summary, in the diagnosis of osteoporotic lumbar compressive fractures in the elderly, the value of CT diagnosis is significantly better than that of $\mathrm{X}$-ray diagnosis, which has a higher diagnostic accuracy and can be widely used clinically. Our clinical practice shows that PKP treatment of osteoporotic thoracolumbar vertebral compression fractures, compared with traditional treatment methods, has outstanding curative effect, short operation time, high safety, and convenient operation. It is a kind of treatment for bone with effective minimally invasive surgery for loose thoracolumbar compression fractures. However, because we have not carried out this work for a long time and the follow-up time of patients is short, we should also find an effective method for the root cause of the patients' disease, so as to find a more complete longterm target treatment plan.

\section{Data Availability}

No data were used to support this study.

\section{Conflicts of Interest}

The authors declare that they have no conflicts of interest.

\section{References}

[1] L. Meccariello, V. F. Muzii, G. Falzarano et al., "Dynamic corset versus three-point brace in the treatment of osteoporotic compression fractures of the thoracic and lumbar spine: a prospective, comparative study," Aging Clinical and Experimental Research, vol. 29, no. 3, pp. 443449, 2017.

[2] R. Mattie, K. Laimi, S. Yu, and M. Saltychev, "Comparing percutaneous vertebroplasty and conservative therapy for treating osteoporotic compression fractures in the thoracic and lumbar spine," Journal of Bone and Joint Surgery, vol. 98, no. 12, pp. 1041-1051, 2016.

[3] H. B. Qu, P. J. Tong, W. F. Ji, and J. Li, "Percutaneous kyphoplasty for the treatment of osteoporotic vertebral compression fracture with degenerative scoliosis," Zhongguo Gu Shang, vol. 29, no. 1, pp. 38-40, 2016.

[4] S. Chen, Z. Mi, C. An-Yuan, and H. Zi-Feng, "Percutaneous kyphoplasty combined with zoledronic acid infusion in the treatment of osteoporotic thoracolumbar fractures in the elderly," Clinical Interventions in Aging, vol. 13, pp. 853-861, 2018.

[5] X. Yang-Jiang, Z. Suo-Dong, and W. U. Bo, "Clinical observation of duhuo jisheng decoction in staging treatment for 48 cases with senile osteoporotic simple thoracolumbar compression fracture," Chinese Journal of Experimental Traditional Medical Formulae, vol. 29, no. 3, pp. 419-424, 2015. 